\section{Snowflake Selfies Research by Students with Smartphones}

Adapted from "Snowflake Selfies:

A Low-Cost, High-Impact Approach toward Student Engagement in Scientific Research (with Their Smartphones)," by Matthew R. Kumjian (The Pennsylvania State University), Kevin A. Bowley, Paul M. Markowski, Kelly Lombardo, Zachary J. Lebo, and Pavlos Kollias. Published online in BAMS, June 2020. For the full, citable article, see DOI:10.1175/BAMS-D-19-0096.1.

(top left) Student from the course taking microscopic photographs; (middle) Paul Markowski launching a radiosonde during a snow event; and (bottom right) measuring the snow depth on the pan.

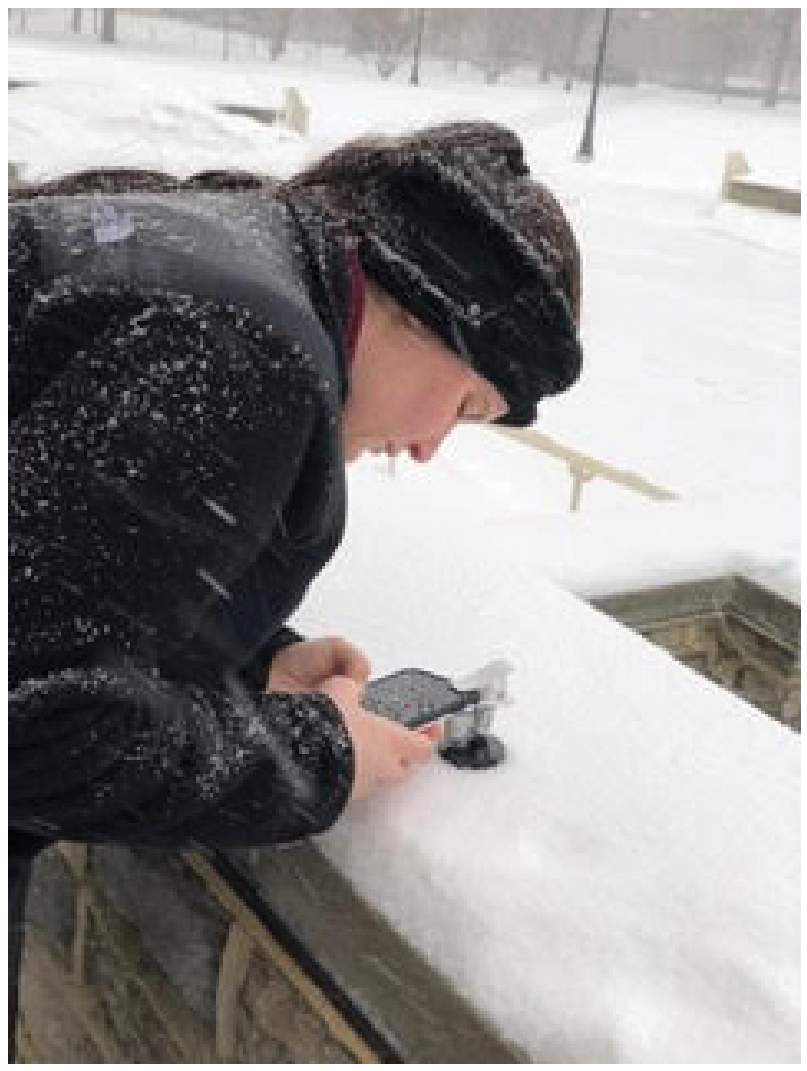

0 ne way to present students with contemporary scientific and societal challenges, incorporating new or emerging technology, is through academic experiences outside the classroom-"engaged scholarship." Engaged scholarship is known to raise student grade point averages (GPAs), increase retention, and improve critical thinking. Examples of engaged scholarship include undergraduate student involvement in research and out-ofclassroom involvement in societal issues of interest to the students.

Snowstorms combine strong student interest with societal relevance. Accurate prediction of snow-to-liquid ratios (SLRs) requires skillful forecasts of vertical air motion, thermodynamic profiles, and microphysical processes, among other factors. Unfortunately, many current microphysics parameterization schemes employed in operational NWP models do not accurately treat snow growth processes, nor do they allow for the natural variability of ice crystal shapes. Thus, an improved understanding of snow microphysics is needed to improve models and reduce uncertainty in snowfall forecasts. 


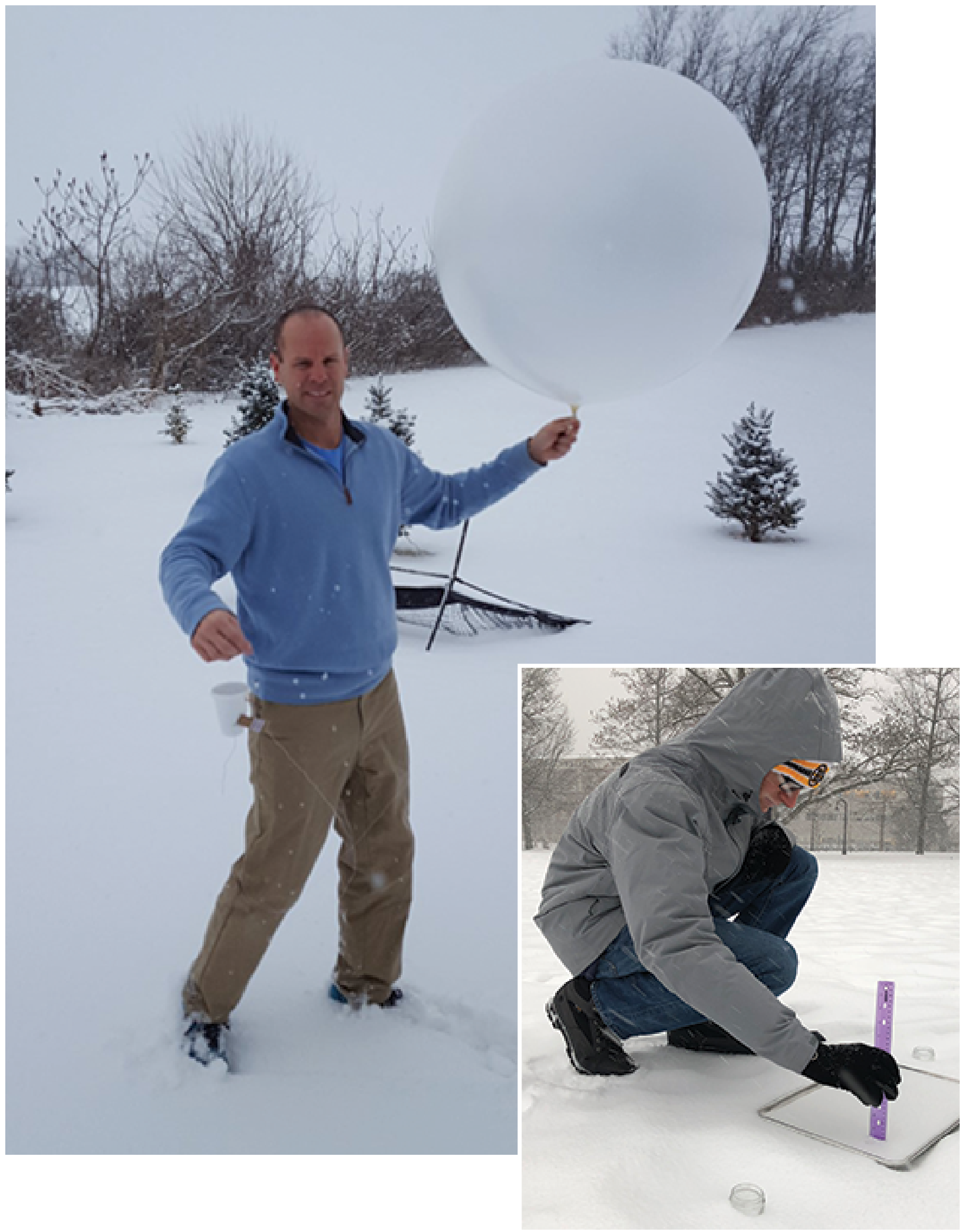




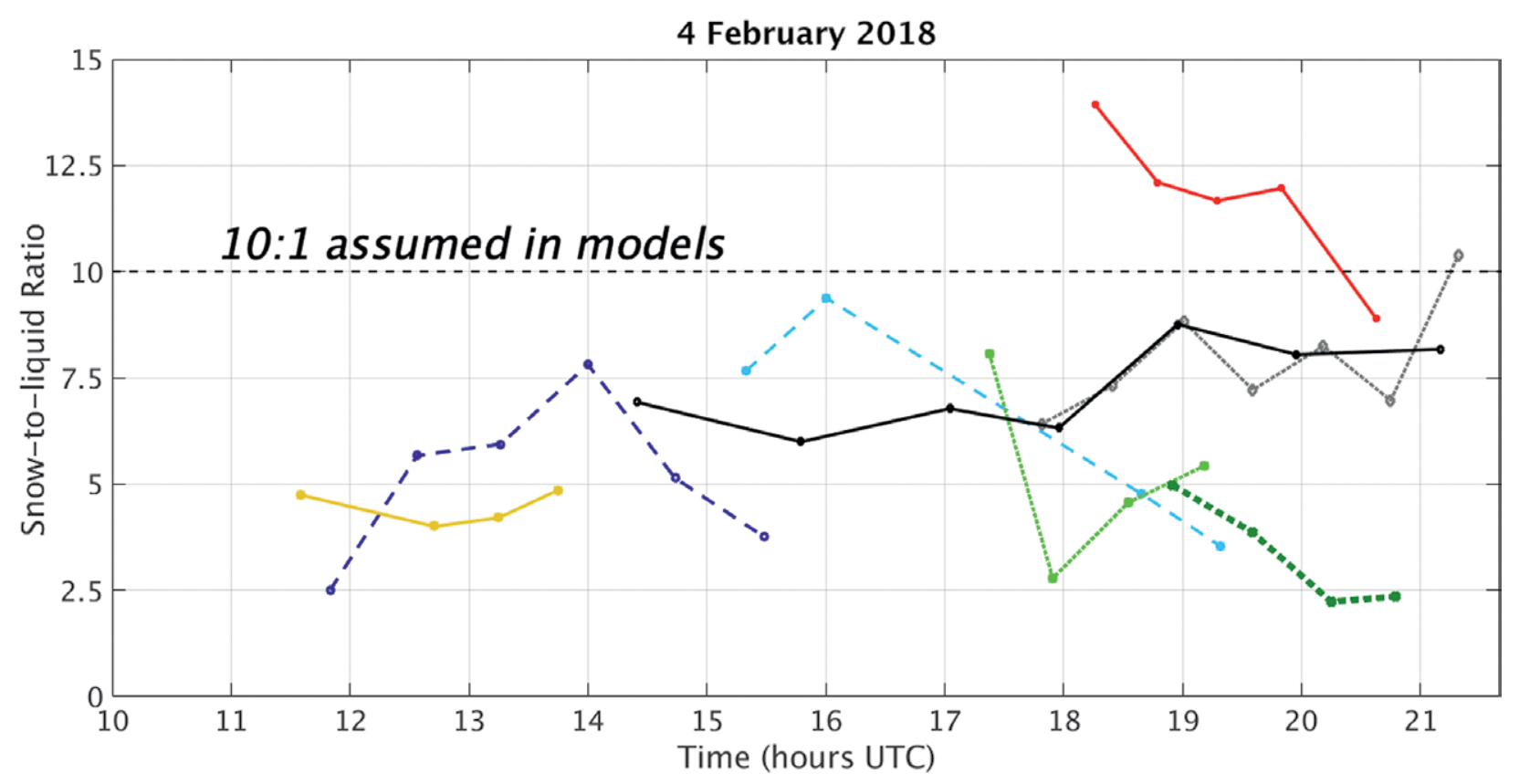

We incorporated snow-related research experiences into undergraduate courses at Penn State to embrace the scientific and societal challenges, our natural wintertime lab-

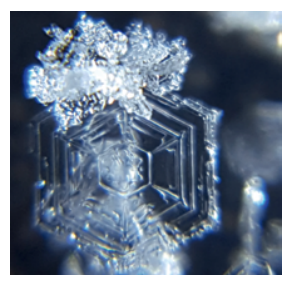
oratory, as well as the much-bemoaned student overuse of smartphones and social media. This "Snowflake Selfies" project was subsequently adapted at the University of Wyoming (which has even longer winters!), engaging a broader population of students in snow science.

\section{Data collection methods}

The equipment used is rather "low tech." Instead of preparing a plywood snow board, each team of students was given a metal baking sheet, two glass jars, a 500-g digital kitchen scale, a ruler, and a thermometer for the project. The metal baking sheet equilibrates to the ambient temperature more rapidly than wood (important when moved outdoors); it is smaller than a snow board, but there is no potential for moisture to affect the snow accumulation or densities in unpredictable ways. Baking sheets with raised edges may affect accumulations, but may be turned upside-down to mitigate these effects.

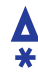

* Time series of SLRs estimated from student measurements on 4 Feb 2018; each line is a different student location.
Each student also received an inexpensive Beileshi clip-on currency microscope (60× magnification) adapter for smartphones, affording much-improved views of riming, hollowing, and small polycrystalline structures. Other options include digital cameras with a "macro" mode setting, and low-cost, USB-pluggable portable microscopes.

The first experiment was performed from 22 January to 11 February 2018 as part of a writing-intensive course that familiarizes in-major undergraduates with taking, analyzing, and communicating a variety of atmospheric measurements. Students learned about snowfall observation and safety guidelines prior to field observations. Each team coordinated their individual intensive observing periods (IOPs) and observation location based on the snowfall outlook, team member availability, and their knowledge of snowfall observation best practices. Then, each student wrote a manuscript-style term

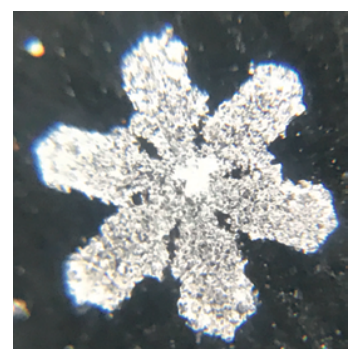
paper presenting their team results. They also made oral presentations in which they further showcased their photos.

For the measurements, students first allowed the baking sheet and jars to equilibrate 

(a)
Also, now getting capped columns and what appear to be some capped bullets! \#SnowflakeSelfies

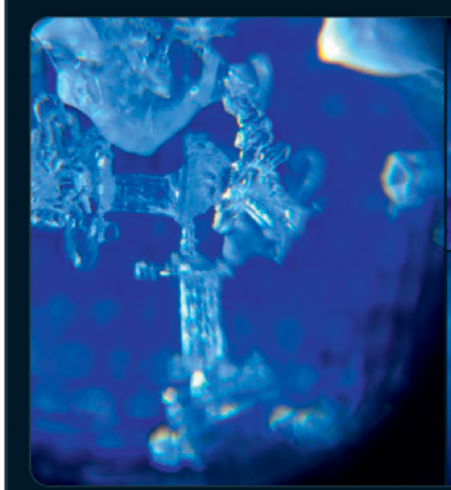

8:52 AM - Feb 7, 2018 - Twitter for Android

3 Retweets $\mathbf{8}$ Likes

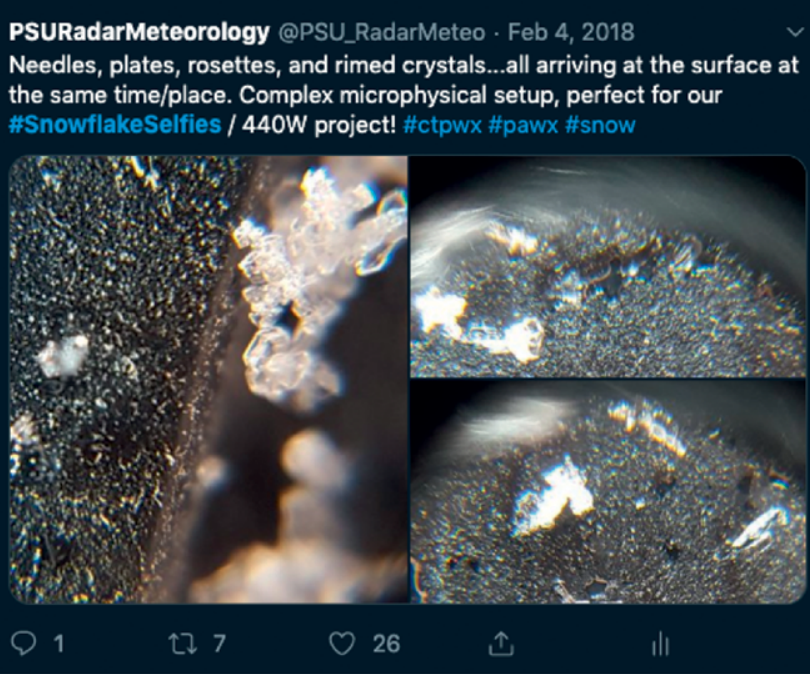

(b)

Some spectacular dendrites falling in State College this morning! The shapes of these crystals tell us a lot about the temperature and relative humidity in the cloud where they formed. One of nature's most beautiful creations @PSU_RadarMeteo
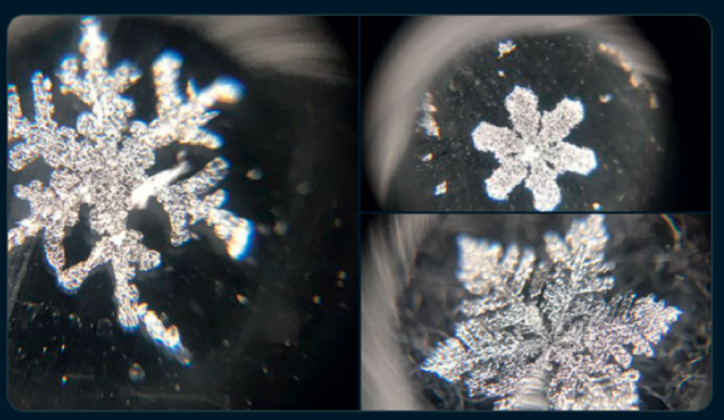

8:48 AM - Feb 6, 2018 - Twitter for iPhone

$\mathbf{7}$ Retweets $\mathbf{4 3}$ Likes

(d) @meteokB

Little bit of everything in State College this morning with the latest set of \#SnowflakeSelfies obs. for @psumeteo 440W @PSU_RadarMeteo
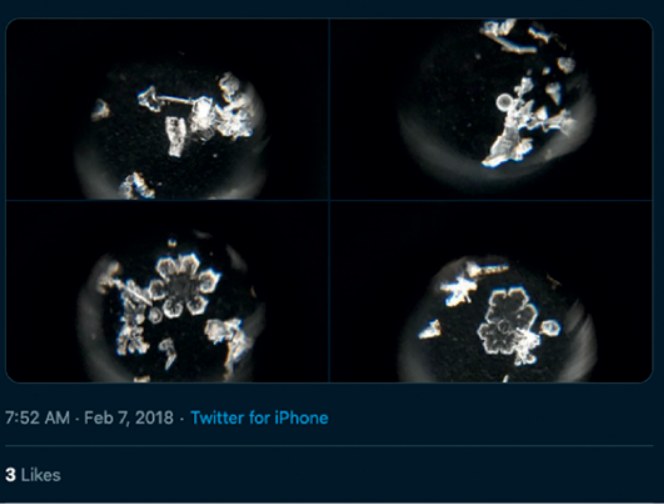

to the ambient temperature outside. The empty jars were weighed to calibrate the mass observations. Students then placed the baking sheet and jars on a level surface to allow precipitation accumulation (usually over 30$60 \mathrm{~min}$ ). They averaged multiple measurements (minimum 5) of snow depth. The snowfall accumulated in the jars was weighed. After measurements, the mass of the empty, dried jars was taken again to estimate the errors associated with the scale and lingering snow in the jar. Using the estimates of snow accumulation and the mass collected in the jars, students could calculate the SLR. The student teams took measurements at least 2-3
* Example social media posts from students and instructors during the Snowflake Selfies project. The names and photos have been removed to protect user privacy. times per event to evaluate the snowstorm's evolution.

Each student group was assigned to find a safe and well-sited observation site using the National Weather Service siting standards, which resulted in a

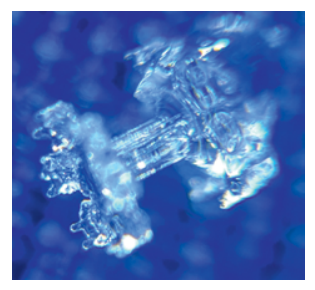
natural geographic dispersion across town, and allowed the spatial distribution of snowfall characteristics to be analyzed as well.

Between accumulation measurements, students used their smartphones with microscope 
adapters to photograph snow crystals in order to characterize the crystal habits and degree of riming. Typically, students would brushclean a surface and allow a few crystals or aggregates to land before taking each picture. Students were eager (and encouraged) to share pic-

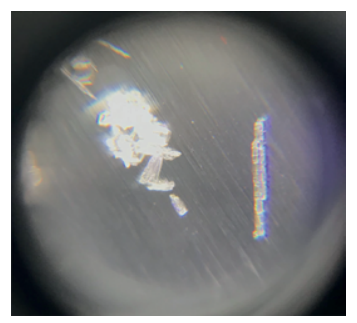
tures on social media.

Stony Brook University's Micro Rain Radar (MRR), a small, K-band, zenith-pointing, frequency-modulated continuous-wave Doppler radar, operated continuously during the Snowflake Selfies project from a rooftop on campus. Data from the Stony Brook MRR provide bulk microphysical and kinematic vertical structure of the precipitation-a context for the students' observations. For several IOPs, a sounding was also released from southwest State College, Pennsylvania. In addition, the local National Weather Service WSR-88D dual-polarization radar (KCCX) is $19 \mathrm{~km}$ northwest of State College. Dual-polarization observations have proven valuable to qualitatively characterize snow microphysical processes, including vapor depositional growth, aggregation, and riming.

\section{Snowflake Selfies observations}

Despite below-average snowfall during the observing periods, the experiment nonetheless showed promise for crowdsourcing observations of snowfall accumulation and estimates of SLRs. We discuss here an example in which nearly every student team took measurements, on 4 February 2018.

The heaviest precipitation rates, in excess of $4 \mathrm{~mm} \mathrm{~h}^{-1}$, were collocated with a cold front extending from

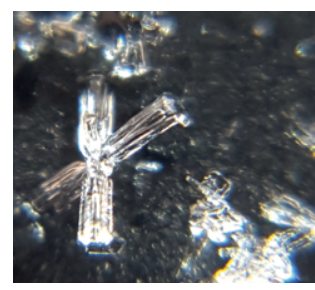
Maryland to Florida. An increase in precipitation intensity was also associated with a moistening of the surfaceto-750-hPa layer near State College, from 1200 UTC to 1800 UTC, eventually resulting in a fully saturated sounding by 2100 UTC-attributed both to moisture advection as well as ongoing precipitation falling through the unsaturated layer.
The clouds, and temperatures below $0^{\circ} \mathrm{C}$, reduced the rate of snowflake sublimation and prevented melting.

Crystals photographed showed a variety of polycrystalline forms, including bullet rosettes. Such habits are known to form at lower temperatures, and are less efficient aggregators than, for example, dendrites or stellars. The lack of significant secondary habits suggests lower supersaturations with respect to ice. At times the particles also showed signs of light riming consistent with radar observations.

The Stony Brook MRR showed increased particle downward motion below $2 \mathrm{~km}$ ARL to values exceeding what is expected for snow, which may arise from riming. Needles and some riming on crystals were captured in photographs.

The small, polycrystalline structures and/ or rimed particles photographed and inferred from radar observations, paired with their intrinsic inefficiency of aggregation, would suggest lower SLRs. The vast majority of teams estimated SLRs smaller than 10:1, in agreement with the expec-

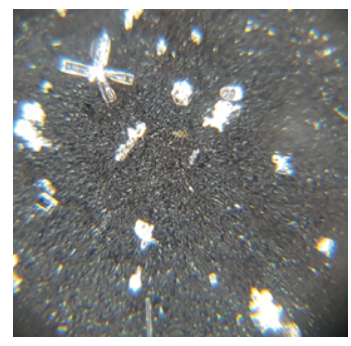
tations based on the snow particles observed. The average, between 5:1 and 8:1 for the duration of the event, is consistent with the relatively homogeneous precipitation depicted on radar imagery. Despite some spread in the resulting SLR estimates between groups, the overall uniformity between groups across the observational domain and the expectations based on the photographs is reasonable and suggests a successful data collection effort.

\section{Educational impact}

Students and instructors frequently posted their photographs and analysis of snow crystals on social media outlets, occasionally using the hashtag "\#SnowflakeSelfies." This provided a pathway toward enhanced engagement with the broader meteorological community and public: several posts registered $>3,000$ views. Social media also provided a means toward communicating important real-time information to local forecasters. 
In one storm with rapidly changing crystal habits and SLRs, the local National Weather Service forecasters adjusted a snow forecast based on the observations the students and instructors shared through social media. The student measurements and photos were taken at a much higher frequency than typical operational observations.

Students faced a challenge in balancing the need to take snowfall observations at various times of the day with busy academic (or extracurricular) calendars. One student suggested that a way to circumvent this issue would be to encourage groups to share their obser-

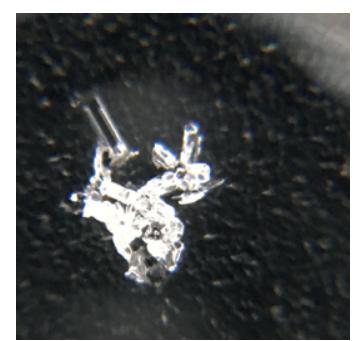
vational data. Similar ideas could be applied if Snowflake Selfies were implemented as a citizen-science project, where daily schedules of observers may not allow for consistent availability for IOPs.

Despite some challenges posed by the weather and below-average snowfall season, we feel the project was a success. The student responses to a postcourse survey were overwhelmingly positive, highlighting effectiveness of the project in engaging students and increasing their enthusiasm for the semester-long project. Use of low-cost but current technology appeared to help stimulate student engagement in the course project.

The natural link to social media helped broaden the engagement to the community level. Based on the success of the experiment, the Penn State Department of Meteorology and Atmospheric Sci-

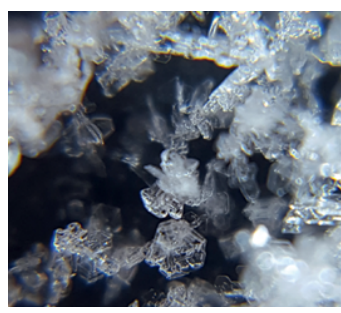
ence purchased for $\$ 500$ a dedicated set of Snowflake Selfies equipment for a class size of 32 students. We encourage other institutions to adapt or implement Snowflake Selfies or similar projects that offer engagement with societally relevant science as well as current technology.

\section{三 METADATA}

BAMS: What would you like readers to learn from this article?

Matthew Kumjian (The Pennsylvania State University): Low-cost, accessible equipment can be used to engage students in meteorological observations and research, and connect them with the natural beauty of snow crystals. Our successful implementation of this type of project demonstrates its ability to increase student interest and involvement in courses, and provides a means to engage the broader community through social media and citizen science.

BAMS: What inspired Snowflake Selfies?

MK: Snow has always provided me with a source of natural wonderment. Finding a relatively accessible way to spread that wonderment, and provide much-needed data, was the inspiration. I have to thank Randy Chase (UIUC) for first showing me the microscope clip-on adapters, which opened the door to this type of project!

BAMS: What surprises/surprised you the most about this project?

MK: The relative ease at which we can obtain striking images of snow crystals and share them with the broader community. The overwhelmingly positive responses to the images shared on social media during the project were encouraging (and pleasantly surprising, these days), too.

BAMS: What was the biggest challenge you encountered while doing this work?

MK: It didn't snow very much in State College during the project (or the follow-up season that we tried it, too). I think the atmosphere is out to spite us.

BAMS: What's next? How will you follow up?

$\mathbf{M K}:$ We will continue to improve the methods and implementation with our experiences and with our students' feedback. We would also like to expand the project to the local community, to engage more of our neighbors in citizen science. 


\section{Be prepared, ' not scared.}

/

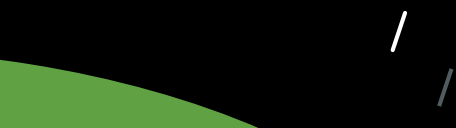

(1)
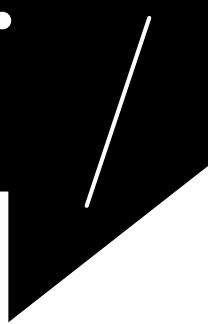

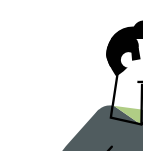
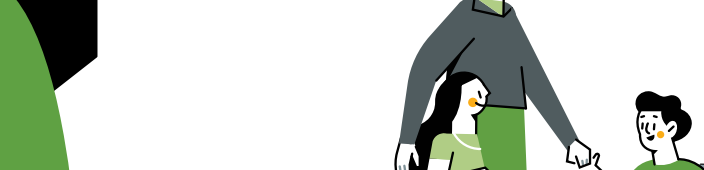

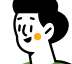
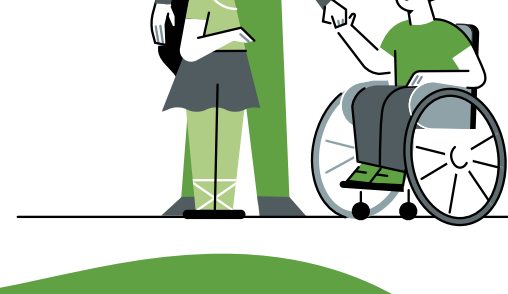

Considering the number of disasters that have occurred in the past decade, chances are you'll experience some sort of emergency. Between school, sports and other activities, chances are you won't be with your kids when it happens.

Ready.gov/kids has the tools to make preparing easy and even fun, so your kids can feel...

Prepared, not scared.

Go to www.Ready.gov/kids and talk to your family today.<smiles>[R10]C1CCC1[R19]</smiles>

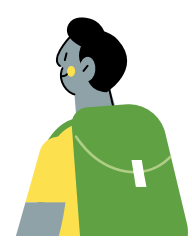

